\title{
A Conceptual Study of Imaging System for 1U CubeSat Application
}

\author{
Fatimah Zaharah Ali, Abhas Maskey, Ahmad Shaqeer Ahmad Thaheer, Wan Ahmad Khusairi Wan \\ Chek and Mohamad Huzaimy Jusoh
}

\begin{abstract}
U CubeSat has limitations in mechanical and electrical properties that pose restriction in selecting imaging sensor for camera mission. As a result, $1 \mathrm{U}$ CubeSat usually carries unfavorable-performance imaging system that delivers low quality of imagery in terms of resolution and other traits. As $1 \mathrm{U}$ CubeSat is mostly suitable for small satellite projects due to shorter development time and cheaper production cost, it is imperatively useful if the $1 \mathrm{U}$ CubeSat can provide high quality imaging system similar to the camera mission provided by $3 \mathrm{U}$ or larger CubeSats which come with longer development time and greater production cost. In this paper, a conceptual study on designing an imaging system with optimal attributes and performance for $1 \mathrm{U}$-sized CubeSat will be discussed. The parameter to be examined for performance improvement is the ground sampling distance (GSD) which is associated with the application of the focal length. The study uses the quantitative measurements as a validation tool to prove the correlation between focal length of the selected lens and the GSD. Moreover, it can also be used to identify the appropriate type of protocol interface to be used that will affect overall performance of the imaging system This conceptual study will provide new guideline in designing a high performance imaging system for small type of satellite, where it will complement the conventional method of selecting the imagery.
\end{abstract}

Index Terms - GSD, imaging system, $1 \mathrm{U}$ CubeSat.

\section{INTRODUCTION}

$\mathrm{C}$ UBESAT is classified as a small type of satellite under the category of Nanosatellite. The classification and its standard names are based on the satellite's mass and size (See Table I) [1], [2], [3]. CubeSat is the notable square-shaped artificial satellite that was introduced by Professor Emeritus Bob Twiggs from Stanford University, California [4], and his co-researcher, Professor Jordi Puig-Suari from California

This manuscript is submitted on $22^{\text {th }}$ June 2021 and accepted on $29^{\text {th }}$ April 202. F. Z. Ali, W. A. K. Wan Chek and M. H. Jusoh are School of Electrical Engineering, College of Engineering, Universiti Teknologi MARA, 40450 Selangor, Malaysia (e-mail: fatimah_zaharah@uitm.edu.my, khusairi5079@uitm.edu.my and huzaimy@uitm.edu.my).

A. Maskey is with Antarikchya Pratisthan Nepal (Space Foundation Nepal) Maharajgunj, Kathmandu, 44618 Nepal (e-mail: editor@madeinepal.com).A. S. Ahmad Thaheer is with School of Aerospace Engineering, Universiti Sains Malaysia, Engineering Campus, 14300 Pulau Pinang (e-mail: ashaqeer@gmail.com).

1985-5389/C 2021 The Authors. Published by UiTM Press. This is an open access article under the CC BY-NC-ND license (http://creativecommons.org /licenses/by-nc-nd/4.0/).
Polytechnic State University in 1999 [5], for the purpose of education demonstration to the students.

Due to wide acceptance specifically from education institutions, the CubeSat technology has been vastly evolved since 2005 [6]. Shorter period of production with minimal cost have been the main interest for researches to be involved in the project of small satellite development. Since 2013, it was reported that more than 100 CubeSats have been launched to the space [6] and the number is still increasing.

TABLE I

CATEGORY OF SMALL SATELLITE

\begin{tabular}{ccccc}
\hline \hline Class & Category & $\begin{array}{c}\text { CubeSat } \\
\text { Standard } \\
\text { Name }\end{array}$ & $\begin{array}{c}\text { Mass } \\
(\mathrm{kg})\end{array}$ & Size $(\mathrm{cm})$ \\
\hline Small & Nanosatellite & $1 \mathrm{U}$ & $1-1.33$ & $10 \times 10 \times 11.35$ \\
& & $2 \mathrm{U}$ & $2-2.66$ & $10 \times 10 \times 22.70$ \\
& $3 \mathrm{U}$ & $<4$ & $10 \times 10 \times 34.05$ \\
& Microsatellite & $6 \mathrm{U}$ & $<12$ & $20 \times 10 \times 34.05$ \\
& & $12 \mathrm{U}$ & $<24$ & $20 \times 20 \times 34.05$ \\
& & $27 \mathrm{U}$ & $<40$ & $10 \times 10 \times 11.35$ \\
\hline \hline
\end{tabular}

From Table I, 1U CubeSat is the smallest Nanosatellite with standard physical dimension of 10 by 10 by $11.35 \mathrm{~cm}$ and weight of 1 to $1.33 \mathrm{~kg}$. These mechanical properties were standardized by the CubeSat's abovementioned founders under the CubeSat Design Specification (CDS) and used as the global reference for CubeSat development. The limitations on the design structure contribute to the limitation on the electrical property where $1 \mathrm{U}$ CubeSat could only provide power ranging from $1 \mathrm{~W}$ to $2.5 \mathrm{~W}$ to its overall systems. These limitations have affected the selection of mission and the components to be used for the $1 \mathrm{U}$ CubeSat.

\section{A. Imaging System of a $1 U$ CubeSat}

Camera mission is the most common onboard mission of any type of satellites. For $1 \mathrm{U}$ CubeSat, the camera mission usually focuses on the earth imaging instead of the remote sensing application. This is due to the satellite's constraints on the mass, size, and power supply that restrict its functionality to have high precision imagery.

Camera with high resolution, where the number of array size is greater, consumes more power for its system's operation. Production of fine imagery from high altitude image capturing activity will require the camera to be equipped with complex 
circuitry. Usually, this type of camera is bulky and heavy in which it is not suitable for the standard design specification of $1 \mathrm{U}$ CubeSat. Besides, the fine imagery utilizes bigger size of data where it will affect the onboard operation and downlink process, as well as the requirement of flash memory to store the captured imageries. Therefore, the selection of camera for imaging mission must not only consider the mechanical and electrical properties of the CubeSat, but also the effect it has on the overall subsystems of the spacecraft.

An imaging system of a satellite commonly consists of few main components such as the imaging sensor, lens including its holder, camera's microcontroller (CAM MCU), flash memory (FM), and an additional filter if necessary. For $1 \mathrm{U}$ CubeSat, the selection of these components must also be based on the electrical and mechanical properties of the spacecraft. Due to the limitations posed by $1 \mathrm{U}$ CubeSat, the selected imaging sensor for the camera mission usually has low precision in terms of resolution.

\section{B. High Performance Imaging System for $1 U$ CubeSat}

Resolution basically describes the quality of the imagery. With higher resolution, a finer image could be obtained from the camera. When a high precision camera module is unable to fit within the limitations of the $1 \mathrm{U}$ CubeSat, the high performing imaging system can be replaced by changing certain parameter of the imaging system in providing better ground resolution. In remote sensing applications that involve the satellite technologies, ground resolution is technically called as ground sampling distance (GSD).

Camera resolution is one of the technical parameters of the imaging sensor that cannot be changed for performance improvement. However, the property of the imagery can still be affected by the implementation of the lens on the imaging sensor. In [7], the GSD may be improved by varying the focal length of the imaging sensor based on the equation in (1), where $H, f$, and $d$, are the altitude in meter (m), focal length in millimeter ( $\mathrm{mm}$ ), and pixel size in $\mathrm{mm}$, respectively. From (1), it is proven that the focal length is one of the crucial parameters in selecting the suitable imaging sensor in order to obtain a refined image with small GSD value for high altitude remote sensing system.

$$
G S D=\frac{H}{f}(d)
$$

The association between the lens and the imaging sensor in term of focal length is further discussed in the next section.

\section{Ground Resolution}

Resolution is often the main parameter to indicate the quality of the imagery. In an imaging system, the resolution specification of an imaging sensor or imager is the number of pixels available to display the captured image. The number of pixels is typically is expressed by an array size. A higher number of pixel available in an image means that a more precise image is produced. This is because, each of the small pixel indicates the reflected electromagnetic (EM) wave of the targeted area.

Ground resolution, on the other hand, is the pixel size of an image that is measured on the ground. Ground resolution can also be referred as spatial resolution or GSD. With the common meter (m) unit, ground resolution will be varied based on the array size or number of pixels of an imagery. High resolution of an imagery means the value of the ground resolution is small. This small value of ground resolution represents the size of a pixel of the image. The smaller the size of the pixel, the higher the number of pixels of an image.

Some of the sensor specification does not show the pixel value in mega pixels (MP) but instead shows it in terms of size of a pixel. Size of a pixel contributes similar attribute as the resolution and number of pixels. Larger pixel size leads to larger area to be seen in an image. If the targeted object is larger than the size of the pixel, the image of the object can be cearly seen on camera. Thus, appropriate imaging sensor's parameters must be evaluated in choosing the suitable camera for onboard imaging system.

\section{THEORITICAL MEASUREMENT}

The quality of an imagery depends on the parameters of the applied imaging system such as imager's array size and distance between the imager and the targerted area. The parameters of the imaging sensor cannot be altered as they are manufactured to distinguish the capability of the sensors. For satellite application, particularly the CubeSat, the distance between the imaging system and the targeted area is also impossible to be changed, as the satellite would require complex system to do so with larger power consumption and larger physical volume of satellite structure (propulsion control mechanism). Therefore, the ray bender, which depends on the lens focal length, might be the only parameter that can be modified for improving the performance of the imaging system, specifically in the case of 1U-sized CubeSat.

In this section, the mathematical quantitative computation will be implemented in order to determine better GSD for $1 \mathrm{U}$ CubeSat with selected compatible lens and imager. This theoretical measurement will be a quantitative assistance in the selection of main components for development of onboard imaging system. However, before the quantitative computation is applied, it is necessary to acquire a suitable imaging sensor or imager for the camera mission system of the $1 \mathrm{U}$ CubeSat. As previously mentioned, $1 \mathrm{U}$-sized CubeSat possesses mechanical and electrical constraints that requires meticulous consideration in selecting components or devices for its application. Thus, the following sub-section will brief the essential requirements to be referred to in selecting an imager for $1 \mathrm{U}$ CubeSat. The requirements are in accordance with the mechanical and electrical attributes of the $1 \mathrm{U}$ CubeSat.

\section{A. Selection of Imager for $1 U$ CubeSat}

Imaging sensor or imager must be $5 \%$ less than the mass of the $1 \mathrm{U}$ CubeSat [8]. This is to ensure that the overall bus system and mission board including the controller can be implemented 
onboard of the satellite without compromising the standard mechanical properties of $1 \mathrm{U}$ CubeSat. In order to simplify the selection process of an imager for $1 \mathrm{U}$ CubeSat, the physical measurements of the camera breakout, specifically the size or the dimension (width $\mathrm{x}$ depth $\mathrm{x}$ height) of the imager, must also be $5 \%$ less than size of $1 \mathrm{U}$ CubeSat. The result of the computed physical requirement is listed in Table II.

TABLE II

MECHANICAL REQUiREMENT OF AN IMAGER For 1U CUBESAT

Max. Size (w x d x h) Range of Mass

$5 \mathrm{~mm} \times 5 \mathrm{~mm} \times 5.675 \mathrm{~mm}$

$50-66.5 \mathrm{~g}$

By using the values in Table II to determine the physical dimension, the selection of an imager for $1 \mathrm{U}$ CubeSat will be straightforward. Once the dimension is determined, the selection process of imager for $1 \mathrm{U}$ CubeSat can focus on the next parameter - an imager that offers high number of array or camera resolution.

Nevertheless, another crucial requirement to be considered during the selection of an imager is power consumption. By referring to the imager specification, the power consumption of the device can be obtained. This value is essential for power budget calculation in order to determine the selected imager is suitable for 1U CubeSat's electrical property.

Another requirement to be considered in selecting an imager for a CubeSat is the imager's interface protocol. However, this solely depends on the design of backplane implemented for the integration of CubeSat's bus systems. For example, if the standard UWE-3 interface is applied, there will be some limitation on interface protocol. UWE-3 is a concept of UNISEC interface designed by University of Würzburg, Germany [9], [10]. The UWE-3 interface has no designed slot for Universal Serial Bus (USB) protocol. As a result, a sensor or device with USB connector cannot be used as the payload for the CubeSat. Besides, the use of USB interface is not preferable for the small category of satellite since this type of satellite suffers higher vibration effect as compared to the traditional satellite during testing and the rocket launching.

Despite of the limited interface protocol issue, the UWE-3 backplane interface board has been adopted for CubeSat development in order to minimize the period of development and assembly processes which also led to minimal incurred costs and fast delivery time [11], [12]. In [13], the 50-pin connectors interface of UWE-3 is defined as the standardized backplane that complies with entire controller and reduce the risk of human errors.

Due to its heritage in space, UWE-3 was also adopted in 1U CubeSat development program by Kyushu Institute of Technology (Kyutech), Japan [14]. Kyutech has initiated the $1 \mathrm{U}$ CubeSat development and launching program that is called as The Joint Global Multi-Nation Birds Satellite Project (BIRDS) since 2015 that involves many developing countries around the world as the members of the program [15]. Through the program that applies the UWE-3 standards as the CubeSat backplane interface, many constellations of $1 \mathrm{U}$ CubeSats have been successfully launched and operated. Thus, this paper is also referring to the BIRDS $1 \mathrm{U}$ CubeSat, as a reference for the conceptual improvement on the imaging system mission. The further discussion on this will be elucidated in the next section of Conceptual Analysis.

The summary of requirements in selecting the imager for a $1 \mathrm{U}$ CubeSat that have been discussed so far is shown below. These important requirements are to be used in finding the suitable imaging sensor for the earth imaging mission of $1 \mathrm{U}$ CubeSat.

- Mass of imager is $5 \%$ less than mass of $1 \mathrm{U}$ CubeSat [5].

- $\quad$ Size of imager is $5 \%$ less than size of $1 \mathrm{U}$ CubeSat.

- Imager must consume low power. Imager's power usage is required to be included in power budget calculation.

- Type of electronic communication protocol used for data transmission or simply the interface. This is usually based on the implemented backplane board.

By following the requirements above, it will be easier to select suitable imager and the options of selection can be narrowed down. These requirements must also consider the camera resolution or array size in order to ensure better performance imager in terms of imagery quality. Once the imager is selected, the quantitative measurement can be performed by referring to imaging sensor's specification and applying appropriate equations.

\section{B. Quantitative Computation}

Based on equation (1), the ground resolution can be computed with the presence of $H, f$, and $d$. The longer $f$ will contribute to small value of GSD that denotes the high ground resolution or high spatial resolution. For CubeSat application, $H$ is a constant value of the altitude where the spacecraft will orbit the earth, while $d$ is determined from specification of the selected imaging sensor or imager. Table III shows the difference of GSD when different values of $f$ is applied. In this Table III, three (3) designs are presented in order to show the relationship between GSD and $f$. The value of $d$ is constant for all three designs since same imaging sensor is applied. In Table III, $d$ will be $1.4 \mu \mathrm{m}$. The altitude $H$ is set to be $400 \mathrm{~km}$, similar to height of International Space Station (ISS) from the earth.

TABLE III

COMPUTED PARAMETERS For THREE DIFFERENT FOCAL LENGTH

\begin{tabular}{cccc}
\hline \hline Parameter & Design 1 & Design 2 & Design 3 \\
\hline Altitude, H & \multicolumn{3}{c}{$400 \mathrm{~km}$} \\
\hline $\begin{array}{c}\text { Sensor Size } \\
\text { (W x H) }\end{array}$ & $3.6 \times 2.7 \mathrm{~mm}$ & \\
\hline $\begin{array}{c}\text { Pixel Size } \\
\text { (W x H) }\end{array}$ & $1.4 \times 1.4 \mu \mathrm{m}$ & \\
\hline Focal Length, f & $15 \mathrm{~mm}$ & $25 \mathrm{~mm}$ & $35 \mathrm{~mm}$ \\
\hline GSD & $37 \mathrm{~m}$ & $22 \mathrm{~m}$ & $16 \mathrm{~m}$ \\
\hline FOV & 14 & 8 & $61.5 \mathrm{~km}$ \\
\hline HFOV & $96.8 \mathrm{~km}$ & $58.1 \mathrm{~km}$ & \\
\hline \hline
\end{tabular}

In Table III, FOV stands for field of view. FOV provides the viewing angle of the sensor as depicted in Fig. 1. HFOV is the horizontal field of view that is measured in kilometer $(\mathrm{km})$. HFOV indicates the length of the earth area horizontally 
covered by the imaging system. Anything that is situated inside the coverage area will be captured by the camera payload from the space. Both FOV and HFOV are calculated using equations (2) and (3), respectively.

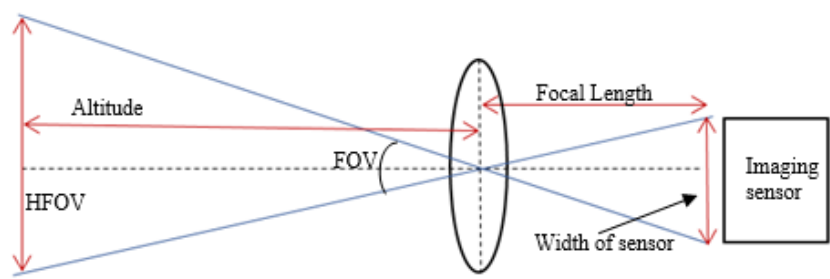

Fig. 1. Focal length of lens, altitude of satellite, and width of camera affect the FOV and HFOV.

$F O V=2 \times \tan ^{-1}\left(\frac{\text { width of sensor }}{2 \times f}\right)$

$H F O V=2 \times H \times \tan \left(\frac{F O V}{2}\right)$

Further discussion in proving the association between the lens and the imaging sensor in terms of focal length is shown in the next section of Conceptual Analysis.

\section{Lens for Improvement of Imager's Performance}

Imaging sensor is the device that holds the lens and filtering system in its architecture. Since the resolution of the imaging sensor for the $1 \mathrm{U}$ CubeSat application are limited, the function of the applied lens can be utilized to modify the quality of an image. As calculated in Table III, varying the length between the imaging sensor and the lens will affect the FOV, and subsequently the resolution of an image on the ground (GSD). The improvement of GSD can be achieved by increasing the focal length. However, the coverage area of the imaging will be reduced. If the improvement of imagery is more on the image quality or clarity, the FOV manipulation can be disregarded since 1U-sized CubeSat poses significant limitations on its mechanical and electrical properties. Likewise, applying camera system with better performance could be impossible due to the presented constraints. Thus, focal length will be one of the parameters that can be used to improve the performance of the camera mission of $1 \mathrm{U}$ CubeSat.

From equation (1), in order to obtain required GSD, it is imperative to find the lens that can support the computed focal length for the imager. Most importantly, the selection of lens must follow the optical specifications of the selected imager. This is to ensure that the lens can be fitly mounted on the imager, and therefore perform light capturing and focusing on the imaging sensor without affecting the quality of the imagery.

Lens is one of the important components of a complete imaging system, besides the camera module. Lens is required to create a focal point and direct the captured light into the image sensor, making it easier for the imaging sensor to focus on the area of interest. Lens holder, on the other hand, is required if the optical lens comes solely without a holder to be mounted on the imaging sensor. The lens must fit within the or greater than the optical area of the imaging sensor in order to avoid the optical vignetting on the image, where some part of the image outside of the lens will be dark.. The vignetting effect will reduce the quality of the captured image. Fig. 2 shows different formats of lens on similar optical size of imaging sensor. This proves that the lens must follow the format indicated on the imager's technical specification. Common lens formats comprise of 1 ", 1/2", 1/3", 1/4", which are measured diagonally onto the lens optical. If the selected imager uses lens format of $1 / 3$ " with stated mount type, the lens must be chosen with similar or greater format with identical mount. Lens's mount is the type of optical connection of the imaging sensor. By choosing the correct mount type, the lens can be attached on the imager. However, a mount adapter can also be implemented on the imaging system if the options for lenses are limited for particular focal length. Total mass of the lens including its holder and the optional mount adapter (if necessarily to be applied) must be counted in the imaging system for $1 \mathrm{U}$-size Cubesat in order not to exceed overall mass budget. To conclude, in selecting compatible and appropriate lens for the chosen imager, it is essential to consider the optical format of the imager, mount type of the imager, and also the supported focal length as required to improve GSD.
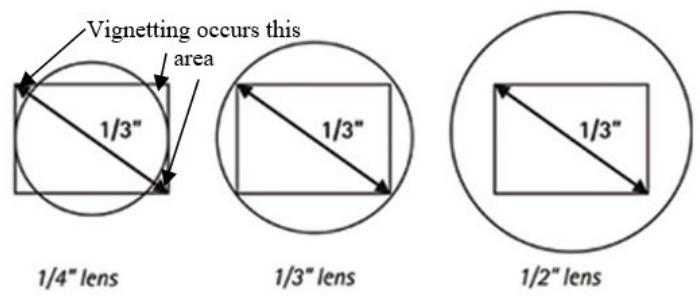

Fig. 2. Selection of lens format must be equal or greater than $1 / 3$ " of imaging sensor's format. Source from [16].

\section{CONCEPtual ANALysis}

The quantitative measurements of GSD and FOV of a selected imaging sensor can be used to preliminarily demonstrate the performance of the imager with its compatible lens for a long working distance, which in this case is the distance between the Earth surface and the orbit where the satellite is revolving. The quality of the imagery output can be theoretically enhanced by calculating the GSD with particular lens focal length that suits the selected imager. The quantitative measurements can also be used as a guideline to select an appropriate imaging sensor and lens for the application of $1 \mathrm{U}-$ size CubeSat. Fig. 3 shows the summary of steps in selecting the appropriate imaging sensor or imager and compatible lens for the camera mission of a $1 \mathrm{U}$-size CubeSat. This includes the complementation for the common process of selecting the imager for $1 \mathrm{U}$ CubeSat as a conceptual study of imaging system design. The common process of imager selection was taken from the process implemented by previous researches in BIRDS program as reference. 


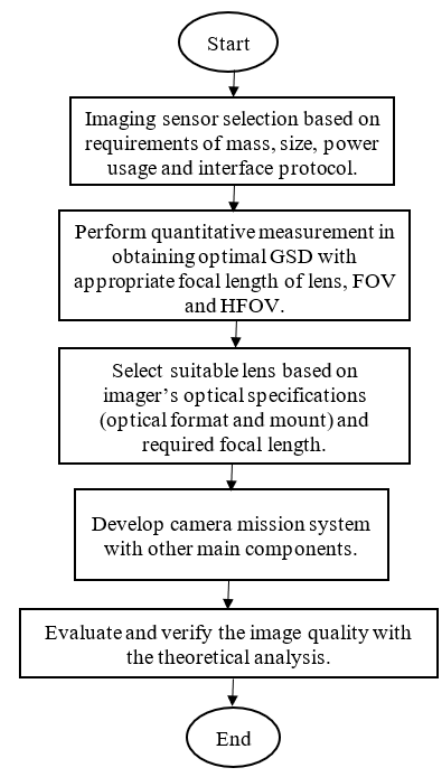

Fig. 3. Process of selecting imaging sensor and lens for an imaging system.

This paper shows that the performance of the imaging system can be improved in terms of the imagery quality by using the quantitative measurement technique. This performance parameters can be evaluated and compared with the hardware development of the camera mission system.

\section{A. Image Quality}

Data in Table III proves that by increasing the value of the focal length of the lens, the GSD value can be made smaller. Small value of GSD indicates that the spatial resolution (the resolution of the image on the ground) is improved and more detailed imagery can be obtained. This is because the viewing angle or FOV value is smaller and that leads the imaging sensor to focus on specific area with swath value or HFOV is only approximately $41.5 \mathrm{~km}$ with focal length of $35 \mathrm{~mm}$. These values are valid for an imager of $5 \mathrm{MP}$ and $14 \mu \mathrm{m}$ pixel size working at $400 \mathrm{~km}$ distance. Each of the pixels offered by the imaging sensor will represent the small area of the imagery. For wider HFOV, the same amount of pixels will be used but the pixels will represent larger targeting area in which the small element cannot be seen clearly.

Fig. 4 - 6 show the relationship between the focal length, HFOV and GSD through tabulated data on the graphs. The graphs are developed based on the quantitative measurement for different values of focal length. The focal length values are randomly selected, as shown on the graph of Fig. 4 and Fig. 5, in order to see the relationship between using shorter focal length and longer focal length. It can be seen that the graphs are exponentially distributed when the focal length in Fig. 4 and Fig. 5, and GSD values in Fig. 6, are increasing. Increasing the focal length will decrease both HFOV and GSD values which is good to provide detailed imagery similar to having a high resolution imaging system. Lowering the GSD, which indicates increasing spatial resolution, will decrease the area covered for imagery.

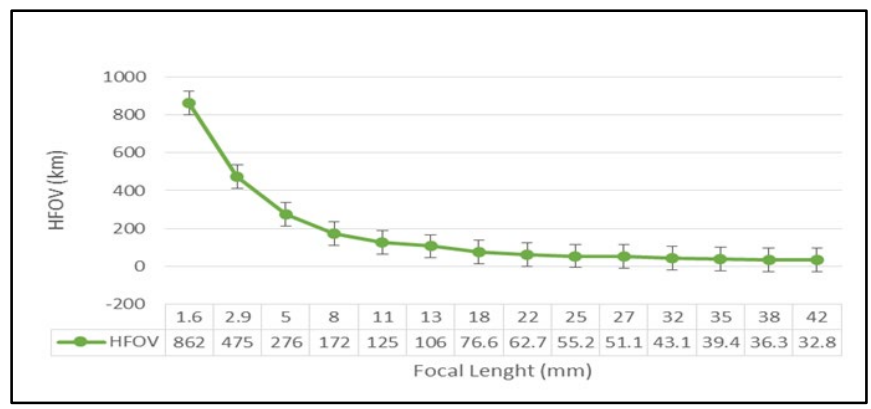

Fig. 4. Graph of HFOV in km versus focal length in $\mathrm{mm}$.

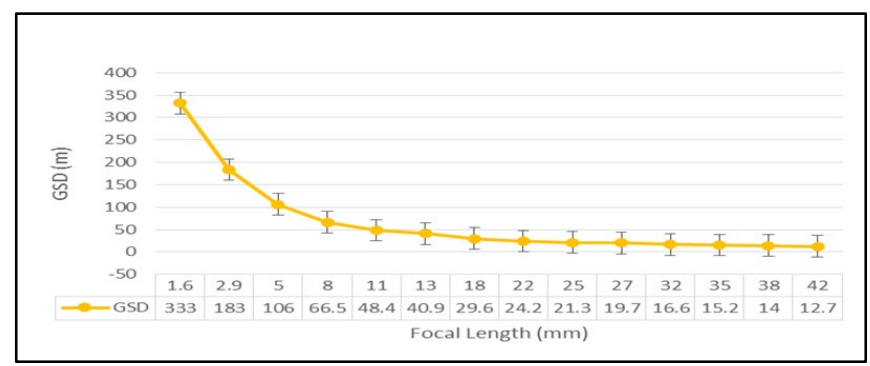

Fig. 5. Graph of GSD in meter versus focal length in $\mathrm{mm}$.

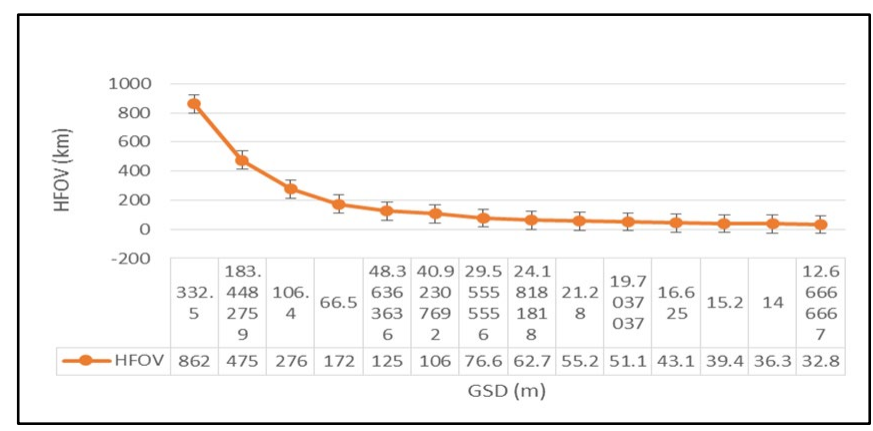

Fig. 6. Graph of HFOV in km versus GSD in meter.

The developed imaging system can be evaluated by comparing the performances based on the discussed parameters above. With the verification, the developed imaging system can be applicable for $1 \mathrm{U}$ CubeSat application with quality of imagery is being focused. In this paper, the obtained parameters from the quantitative measurement were validated by comparing the values with the parameters obtained from the implemented imaging mission by BIRDS program. It was proven through the mathematical analysis that the GSD of the imagery can be improved by increasing the focal length of the applied lens.

\section{CONCLUSION}

This conceptual study of using quantitative measurement of imager's parameters for performance analysis is indeed a state of the art of the technology for satellite application. The imaging system as camera mission for $1 \mathrm{U}$ CubeSat can be improved based on the parameters and performance discussed in previous sections. The study and findings from the quantitative measurement will provide another clear process and guidelines in designing a high performance imaging mission in terms of imagery quality instead of focusing on the high precision attributes with many limitation and constraints 
for future small satellite development.

\section{FUTURE WORK}

This paper provides the guidelines of designing the imaging system with high ground resolution for $1 \mathrm{U}$ CubeSat application. Thus, the quality of the image will be based on the small value of GSD of the captured image. By referring to the guidelines, any imagery that would be captured by the imaging sensor onboard the satellite will have similar value of GSD as calculated through the quantitative measurement.

In the future work, the conceptual study will be used to design the imaging system for the $1 \mathrm{U}$ CubeSat application, and the image captured by the imaging system will be acquired in validating the parameters shown in quantitative measurement.

\section{ACKNOWLEDGMENT}

The authors would like to thank the Ministry of Science, Technology, and Innovation (MOSTI) for the financial support given through the International Collaborative Fund (ICF) 2021. The authors would also like to express the gratitude for the supports and guidance given by Kyutech through the BIRDS Satellite Program.

\section{REFERENCES}

[1] F. Dannemann and M. Jetzschmann, "Technology-Driven Design of a Scalable Small Satellite Platform,” Proc. 4S Symp., no. 1, pp. 1-8, 2016.

[2] Y. J. Song, H. Jin, and I. Garick-Bethell, "Lunar CubeSat impact trajectory characteristics as a function of its release conditions," Math. Probl. Eng., vol. 2015, 2015, doi: 10.1155/2015/681901.

[3] P. Domain, "6U CubeSat Design Specification Rev. 1.0 The CubeSat Program, Cal Poly SLO 6U CubeSat Design Specification Revision 1.0 (CP-6UCDS-1.0) Document Classification X Public Domain 6U CubeSat Design Specification Rev. 1.0 The CubeSat Program, Cal Poly SLO CHANG," vol. 0, pp. 0-26, 2018.

[4] H. Helvajian and S. Janson, "Small Satellites: Past, Present, and Future," Small Satell. Past, Present. Futur., 2009, doi: 10.2514/4.989223.

[5] D. Werner, "Cubesat co-inventor Jordi Puig-Suari sails into the sunset," Space News, 2018. https://spacenews.com/cubesat-co-inventor-jordipuig-suari-sails-into-the-sunset/.

[6] M. Swartwout, "The first one hundred CubeSats : A statistical look," J. Small Satell., vol. 2, no. 2, pp. 213-233, 2013.

[7] Y. Yang, Z. Lin, and F. Liu, "Stable imaging and accuracy issues of lowaltitude unmanned aerial vehicle photogrammetry systems," Remote Sens., vol. 8, no. 4, 2016, doi: 10.3390/rs8040316.

[8] H. Kuuste et al., "Kaamerasüsteem nanosatelliitide lähioperatsioonide jälgimiseks," Proc. Est. Acad. Sci., vol. 63, no. 2S, pp. 250-257, 2014, doi: 10.3176/proc.2014.2S.06.

[9] K. Pradhan, F. Pauline, G. Maeda, S. Kim, H. Masui, and M. Cho, " $\{$ BIRDS-2\}: Multi-Nation \{Cubesat\} Constellation Project for Learning and Capacity Building," Proc. 32nd Annu. AIAA/USU Conf. Small Satell., 2018.

[10] T. Tumenjargal, S. Kim, H. Masui, and M. Cho, "CubeSat bus interface with Complex Programmable Logic Device," Acta Astronaut., vol. 160, no. April, pp. 331-342, 2019, doi: 10.1016/j.actaastro.2019.04.047.

[11] T. Tumenjargal, "Standardized, flexible interface design for a CubeSat bus system," 2019.

[12] T. Tumenjargal, "SSC19-WP1-21 Programmable CubeSat Interface Board to Reduce Costs and Delivery Time."

[13] UNISEC Europe, "CubeSat Subsystem Interface Definition (Proposal)," pp. $\quad 0-10, \quad 2015, \quad$ [Online]. Available: http://uniseceurope.eu/wordpress/wp-content/uploads/CubeSat-Subsystem-InterfaceStandard.pdf.

[14] M. H. Azami et al., "BIRDS-2: A Constellation of Joint Global MultiNation 1U CubeSats," J. Phys. Conf. Ser., vol. 1152, no. 1, 2019, doi: 10.1088/1742-6596/1152/1/012008
[15] T. Joint, G. Multi, N. Birds, and S. Project, “ 「 Joint Global Multi Nation Birds （Birds Satellite Project）,” 2017.

[16] Jon Chouinard, "how-does-a-lens-optical-format-relate-to-machinevision-cameras @ www.1stvision.com,” 2017. https://www.1stvision.com/machine-vision-solutions/2017/08/how-doesa-lens-optical-format-relate-to-machine-vision-cameras.html 